\title{
Substitutionstherapie
}

\section{Zwei neue extrabudgetäre Leistungen zum 1. Oktober}

\author{
Die Substitutionsbehandlung wird durch zwei neue EBM-Positionen \\ aufgewertet: Die „Konsiliarische Untersuchung" und die „Take-Home- \\ Vergabe" werden jetzt eigens und extrabudgetär honoriert.
}

$\mathrm{m}$ Juni hat der Bewertungsausschuss zwei neue Gebührenordnungspositionen (GOP) zur Substitutionsbehandlung beschlossen. Damit werde der Weiterentwicklung der Betäubungsmittelverschreibungsverordnung (BtMVV) Rechnung getragen, heißt es. So gelten ab 1. Oktober 2017 eigene EBM-Ziffern zur Abrechnung des Prüf- und Beratungsaufwandes bei der Verschreibung von Substitutionsmitteln zur eigenverantwortlichen Einnahme („Take-HomeVergabe") sowie für die konsiliarische Untersuchung durch qualifizierte Suchtmediziner. Außerdem kann die Substitutionsbehandlung nicht nur dann im Hausbesuch erfolgen, wenn ein Patient wegen einer anderen Krankheit nicht in der Lage ist, die Praxis aufzusuchen, sondern auch, wenn er pflegebedürftig ist.

\section{Die Beschlüsse im Detail}

_Aufnahme der GOP 01949 in den

EBM-Abschnitt 1.8 für die „substitu- tionsgestützte Behandlung Opiatabhängiger im Rahmen einer TakeHome-Vergabe“. Extrabudgetär werden für diese je Behandlungstag aber maximal zweimal pro Behandlungswoche anzusetzende Ziffer 7,27 € (69 Punkte) vergütet. Daneben ist die Abrechnung der alten GOP 01950 (,,Substitutionsgestützte Behandlung Opiatabhängiger") keineswegs kategorisch ausgeschlossen. „Kommt der TakeHome-Patient öfter (als zweimal pro Woche, Anm. d. Red.) in die Praxis, kann dieser Kontakt bei Vorliegen einer medizinischen Begründung über die GOP 01950 zusätzlich abgerechnet werden“, erläutert die KBV.

_-Aufnahme der GOP 01960, mit der die „Konsiliarische Untersuchung und Beratung eines Patienten" gemäß der Anforderung der BtMVV abgerechnet werden kann. Zur Erläuterung: Suchtmedizinisch nicht eigens qualifizierte Ärzte müssen sich zu Beginn einer

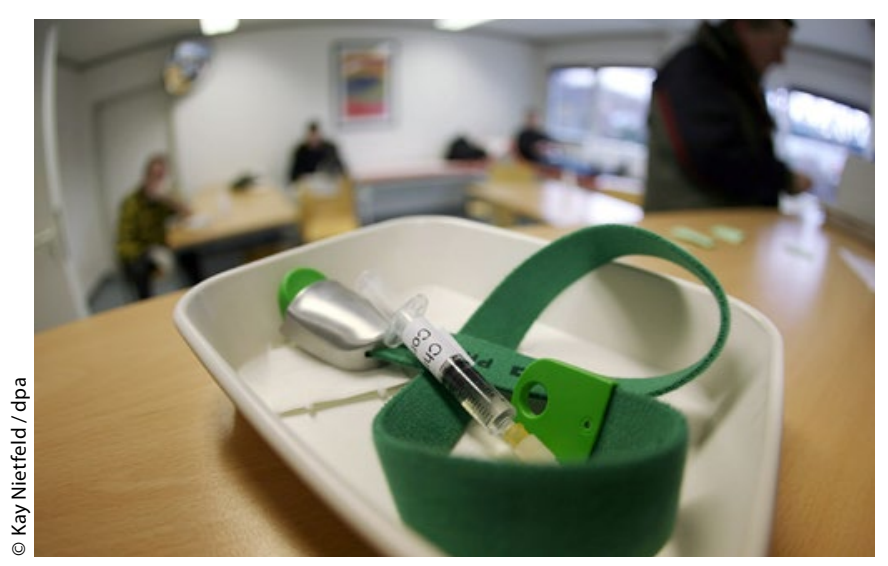

Mit der jüngsten BtMVV-Novellierung dürfen Ärzte ohne zusätzliche Qualifikation jetzt zehn anstelle bislang drei Patienten substituieren.
2.119 Ärzte waren ausweislich des aktuellen Qualitätsberichts der KBV im Berichtsjahr 2015 bundesweit in der Substitutionstherapie tätig und reichten im Schlussquartal entsprechende Abrechnungen ein. Etwas mehr (2.870 Ärzte) besaßen eine Abrechnungsgenehmigung zur Substitutionstherapie. Im Vorjahr 2014 hatten noch 2.961 Ärzte diese Genehmigung. Lediglich neun Praxen nahmen im Berichtsjahr an der Suchtbehandlung mittels synthetischem Heroin (Diamorphin) teil.

Substitutionsbehandlung mit entsprechend qualifizierten Kollegen abstimmen. Dieser Suchtmediziner muss den Patienten wenigstens einmal im Quartal sehen. Laut KBV wurden diese Konsultationen „bislang über eine Grundpauschale innerhalb des Budgets abgerechnet“. Künftig erhalten Suchtmediziner für die konsiliarische Untersuchung im Behandlungsfall extrabudgetär 9,48 € (90 Punkte). Mit dieser neuen GOP werden auch die voraussichtlich "steigende Zahl von Konsiliarverfahren berücksichtigt", denn mit der jüngsten BtMVV-Novellierung dürfen Ärzte ohne zusätzliche Qualifikation jetzt „zehn statt bislang drei Patienten substituieren".

- Mittels einer Ergänzung in der Anmerkung zur GOP 01950 wird jetzt die Möglichkeit eröffnet, Substitutionsbehandlungen auch im Hausbesuch zu erbringen, für den Fall, dass der Patient pflegebedürftig gemäß Einstufung in einen Pflegegrad ist. Zur GOP 01950 können dann die Hausbesuchsziffern 01410 und 01413 (Mitbesuch) angesetzt werden. Gleiches gilt übrigens auch für die Take-Home-Vergabe nach der neuen GOP 01949.

Christoph Winnat 